\title{
The Large-Small Group-Based Evolutionary Game on Knowledge Sharing in Uncertain Environment under the Background of Telemedicine Service
}

\author{
Yuxuan Gao' and Bingzhen Sun ${ }^{1}{ }^{2}$ \\ ${ }^{1}$ School of Marxism, Xidian University, Xi'an 710071, China \\ ${ }^{2}$ School of Economics and Management, Xidian University, Xi'an 710071, China \\ Correspondence should be addressed to Bingzhen Sun; bzsun@xidian.edu.cn
}

Received 11 June 2020; Revised 23 September 2020; Accepted 24 September 2020; Published 8 October 2020

Academic Editor: Michele Scarpiniti

Copyright (C) 2020 Yuxuan Gao and Bingzhen Sun. This is an open access article distributed under the Creative Commons Attribution License, which permits unrestricted use, distribution, and reproduction in any medium, provided the original work is properly cited.

\begin{abstract}
Group decision-making is an effective method to deal with complex unstructured problem in uncertain environment, and it has been widely used in many fields such as medical decision-making. This is a novel study that considers the decision-makers as different groups in the group decision-making problems in uncertain environment. This paper aims to present a novel method combined with evolutionary game for decision-making problem of knowledge sharing in uncertain environment between the large and the small groups in Telemedicine service. For this purpose, the evolutionary game model is constructed to solve decisionmaking problem of large-small group. Through analyzing the evolutionary path and balance, the influencing factors of the selection strategies and objective and subjective factors restricting the establishment of knowledge sharing between the large and small groups cloud be determined. Finally, a numerical simulation experiment is conducted with Matlab to demonstrate the feasibility of the proposed method. In this study, the range of decision groups in the research of group decision-making problem has been expanded, and the complicated factors of knowledge sharing between hospitals in uncertain environment under the background of Telemedicine service have been discussed.
\end{abstract}

\section{Introduction}

Decision-making may take place anytime and anywhere in our life. With the increasing complexity of social-economic environment and the complexity of decision-making in reality, it is unlikely for a single expert to consider all the relevant aspects of a decision-making problem. Therefore, there is a need for increasing the number of decision experts and enlarging the scope of decision experts. Group decisionmaking (GDM) refers to the selection of the best option from a set of alternatives according to the opinions of a number of decision-makers (DMs). Due to the differences in decisionmaking identity, personal experience, and subjective preferences, DMs represent different interests, values, and preferences. Therefore, how to distinguish the DMs of different interest preferences and analyze the factors that influence their decision-making has become a significant issue in group decision-making.

The research on group decision-making has achieved a lot of results. They can be summarized as a direct method and an indirect method. The direct method mainly achieves consensus by defining operators and aggregating computations. Xu developed an error-analysis-based method for the priority of an intuitionistic preference relation [1] and researched a survey of approaches to decision-making with intuitionistic fuzzy preference relations [2]. Yager proposed some of the considerations and information that must be involved in the development of a multisource data fusion algorithm [3] and discussed the role of perception-based granular probability distributions as a means of modeling the uncertainty profiles of the alternatives [4]. The indirect method mainly considers the cost and benefit of consensus, 
the preference of experts, and the environment of expert decision-making. Gong et al. studied the consensus strategy theoretically and practically from the perspective of maximum return and minimum cost and the opinion of interval preference [5, 6]. Wu [7-9] and Liu [10] constructed consensus models based on different factors for group decisionmaking under social network. Group decision-making is also widely applied in various fields: the consensus theory application combined with other uncertainty mathematical theories [11,12], rough set theory [13], game theory [14-16], and other multiattribute decision-making methods [17-19].

As seen from the existing studies, most of the existing group decision-making studies have focused on the general theoretical model and the perfection innovation of mathematical algorithms and pure theoretical aspect, by considering the scheme, preference, weight, etc. The core of the research is how to make the expert preference high consistency, reach a consensus, and form an objective and reasonable scheduling scheme. The existing literature of group decision-making mostly considers the decision-making situation of a single group. Besides, all the identities of DMs in the group are equivalent; that is to say, there is no distinction between the DMs in the group. Practically, in the group decision-making process of many important problems, decision-makers are usually composed of two DGs (decision groups): large group and small group. Moreover, in the large-small group decisionmaking, there is also a conflict of interest, like a game.

Let us introduce the situation of a decision problem that requires DMs of the large-small group to make decisions: knowledge sharing refers to two persons or groups from different organizations exchanging and discussing knowledge through various means and channels, aiming at expanding the use and the effect of knowledge through the exchange of knowledge. As the saying goes, "The truth is in the hands of a few." and so is the knowledge. Therefore, we believe that the higher level of knowledge is in the small group, and the lower level of knowledge is in the large group. The ideal situation is that both groups share knowledge to achieve a win-win situation. However, since the decisionmakers are not entirely rational, they want to pay less and benefit more, so one or both parties may not share knowledge. Therefore, how can we analyze the inner and outer factors that may influence the decision intention of two DGs, give reasonable suggestion and strategy, make the decision of two DGs reach consensus, and achieve win-win result finally?

It is considered that the decisions of large-small group have both cooperative consensus [20] and interest differences. In this paper, the decision-making in knowledge sharing is used as a case to study the influence factors in the decision-making of the large-small group. Since there are prisoners' dilemmas and adverse selection in the decisionmaking process of knowledge sharing between the large and small groups, game theory is the most suitable tool to solve this problem. Game theory, as a new mathematical theory and tool to handle the complexity of decisionmaking problem under uncertainty, was proposed by Von Neumann and Morgenstern in 1944. In the past years, game theory has become the core content in the mainstream in the economics and management field. Moreover, it has almost become the basic analytical tool and study language of economists and management experts in the entire field. So far, it has been successfully applied to many areas: oligopoly games [21], cooperative games [22-24], fuzzy games [25-27], and so on [28, 29]. Therefore, game theory has been widely applied in the study of knowledge sharing. This paper is intended to study this problem by adopting evolutionary game theory. There are already a few studies about the decision-making problem of knowledge sharing between the large and small groups but are still preliminary studies.

Based on this, the game theory is introduced into the decision-making problem of knowledge sharing between the large and small groups under the background of Telemedicine service. The evolutionary model of the selection behavior of the large-small group is established in combination with China's actual conditions. Then, the evolutionary path, evolutionary balance, and influencing factors are analyzed deeply. The influencing factors are simulated, and the corresponding policy suggestions are given at last. This paper studies how to find out the influencing factors of strategies affecting the consensus of large and small groups, carry out evolutionary analysis, and put forward corresponding countermeasures and suggestions. The aim is to essentially make large and small groups more likely to choose strategies that can reach consensus.

The rest of this paper is organized as follows: in Section 2, we make a brief introduction of the decision-making problem of the knowledge sharing between the large and small groups in Telemedicine services and then set the parameters. In Section 3, we establish and solve the evolutionary model of the selection behavior of the large-small group. In Section 4, we apply our approach through a case study. At last, we conclude our research and set out further research directions in Section 5.

\section{The Statement and Assumption of the Group Decision-Making Problem between the Large and Small Groups}

2.1. Problem Statement. In the decision-making problem of knowledge sharing between the large and small groups under the background of Telemedicine service, the main subject of knowledge sharing is the hospitals. It is the typical knowledge organization. With the gradual integration of Telemedicine service into the medical system, the general hospitals in the remote areas could not only solve more medical needs of patients, but also get the help and guidance of specialized hospitals in developed areas to share knowledge. It helps them to improve their own medical technology. Through the medium and platforms of Telemedicine service, hospitals can integrate knowledge resources, speed up knowledge innovation, and improve the efficiency of knowledge innovation through sharing knowledge with each other. Through the sharing mechanism, the mutual transformation of tacit knowledge and explicit knowledge in the organization can be realized, and 
the accumulation, sharing, and innovation of knowledge can be accomplished finally.

Here, we represent specialized hospitals in developed areas with advanced medical technology as the small group in the process of knowledge sharing, and general hospitals in the remote areas with backward medical technology as the large group in the process of knowledge sharing. Therefore, under the background of Telemedicine service, the subjects in knowledge sharing are specialized hospitals in developed areas and general hospitals in remote areas. Generally, general hospitals have more abundant local disease cases and local medical specialty knowledge systems. And specialized hospitals have better medical equipment and medical technology. At the same time, specialized hospitals and general hospitals both have the rights to share knowledge with each other to maximize the benefit of the hospitals. That is to say, there are two strategies for the two groups, namely, to select to share or not to share knowledge. If both groups select not to share knowledge, the end result is that neither group has benefited.

2.2. Parameter Definitions and Assumptions. The variables involved in the game theory of knowledge sharing of hospitals under the background of Telemedicine service are described below:

$E_{g}, E_{s}$ : the normal benefits when general hospitals and specialized hospitals do not share knowledge.

$K_{g}, K_{s}$ : the knowledge level of general hospitals and specialized hospitals. The higher the knowledge level, the greater the benefit the other may gain. Here, we believe that the knowledge of specialized hospitals is significantly higher than that of general hospitals; namely, $K_{g}<K_{s}$.

$\mu_{g}, \mu_{s}$ : the ability of general hospitals and specialized hospitals to absorb and transform knowledge.

$t_{g}, t_{s}$ : the degree of effort that general hospitals and specialized hospitals make to share knowledge.

$\sigma$ : the degree of complementary knowledge of general hospitals and specialized hospitals.

$\lambda_{g}, \lambda_{s}$ : the trust degree of general hospitals and specialized hospitals. Here, we assume that general hospitals have a higher level of trust in specialized hospitals; namely, $\lambda_{g}>\lambda_{s}$.

$C_{g}, C_{s}$ : the cost coefficient of knowledge sharing in general hospitals and specialized hospitals. Here, we assume that specialized hospitals are more at risk of losing their intellectual advantage when they are sharing knowledge, and therefore their costs are higher; namely, $C_{g}<C_{s}$.

$\alpha_{g}, \alpha_{s}$ : the incentive coefficient. The incentives offered by the government when knowledge is shared between general hospitals and specialized hospitals, including funds and policies. Here, we assume that, in the knowledge sharing model under the background of Telemedicine service, the main contributor to knowledge sharing is specialized hospitals. Therefore, the incentives of government to specialized hospitals should be weighted accordingly; namely, $\alpha_{g}<\alpha_{s}$. $\beta_{g}, \beta_{s}$ : the punishment given by the government when general hospitals and specialized hospitals do not share knowledge. Here, we assume that the government should impose more serious punishment for the nonsharing of knowledge by specialized hospitals in order to promote the development of the Telemedicine service; namely, $\beta_{g}<\beta_{s}$.

In the process of the Telemedicine service, general hospitals and specialized hospitals can not only obtain the normal benefits, but also gain the direct benefits and synergies benefits of knowledge sharing. Direct benefits refer to the benefits that the hospitals groups absorb and convert into their own capabilities. They are influenced by the knowledge of the other party and the own knowledge absorption and transformation ability and effort degree. According to the above analysis, in the Telemedicine service, the direct benefits of general hospitals and specialized hospitals obtained in the process of knowledge sharing can be expressed as $\mu_{g} t_{g} K_{s}$ and $\mu_{s} t_{s} K_{g}$. Synergies benefits refer to the benefits brought by the new effects derived by the integration of the original knowledge when the general hospitals and the specialized hospitals are sharing the knowledge. They are influenced by the knowledge level of the other, the degree of knowledge complementation, and trust of each other. According to the above analysis, in the Telemedicine service, the synergistic benefits of general hospitals and specialized hospitals in the process of knowledge sharing can be expressed as $\sigma \lambda_{s} K_{s}$ and $\sigma \lambda_{g} K_{g}$ in the process of knowledge sharing.

The shared incentive obtained by knowledge sharing is related to the amount of knowledge shared. The greater the amount of knowledge shared, the more incentives the government will give. The government incentives should be treated differently because of the different areas in which the general hospitals and the specialized hospitals are located. They are influenced by the amount of knowledge and trust of each other. According to the above analysis, in the Telemedicine service, the shared incentive of general hospitals and specialized hospitals in the process of knowledge sharing can be expressed as $\alpha_{g} \lambda_{g} K_{g}$ and $\alpha_{s} \lambda_{s} K_{s}$.

The process of knowledge sharing takes time and energy, as well as the risk of loss of knowledge advantage. The cost is influenced by the own knowledge level. Therefore, in the Telemedicine service, the costs of general hospitals and specialized hospitals in the process of knowledge sharing can be expressed as $C_{g} K_{g}$ and $C_{s} K_{s}$. Furthermore, in the knowledge sharing, incentives and punishments should only play a supporting role and cannot affect the motivation of hospitals to select knowledge sharing strategies. Therefore, in the Telemedicine service, the incentive and punishment should be less than the costs in the process of knowledge sharing; namely, $a_{g} \lambda_{g} K g_{g}+\beta_{g}<C_{g} K_{g}, a_{s} \lambda_{s} K_{s}+\beta_{s}<C_{s} K_{s}$.

\section{The Methodology and Model of the Group Decision-Making Problem between the Large and Small Groups}

3.1. Model Derivation and Stability Analysis. This paper analyzes the process of hospitals knowledge sharing under 
the background of Telemedicine service by applying "dynamic game with complete information" theory. The benefit function of the groups can be analyzed in several cases:

(1) When general hospitals and specialized hospitals select the knowledge sharing strategy, both groups gain the benefits of knowledge sharing, including normal benefits, direct benefits, synergistic benefits, shared incentive, and costs.

The benefit of general hospitals can be expressed as $E_{g}+\mu_{g} t_{g} K_{s}+\sigma \lambda_{s} K_{s}+\alpha_{g} \lambda_{g} K_{g}-C_{g} K_{g}$.

The benefit of specialized hospitals can be expressed as $E_{s}+\mu_{s} t_{s} K_{s}+\sigma \lambda_{g} K_{g}+\alpha_{s} \lambda_{s} K_{s}-C_{s} K_{s}$.

(2) When the general hospital does not select the knowledge sharing strategy while the specialized hospital selects the knowledge sharing strategy, the benefits of the general hospital include normal benefits, direct benefits, and punishments, and the benefits of the specialized hospital include normal benefits, shared incentive, and costs.

The benefit of general hospitals can be expressed as $E_{g}+\mu_{g} t_{g} K_{s}-\beta_{g}$.

The benefit of specialized hospitals can be expressed as $E_{s}+\alpha_{s} \lambda_{s} K_{s}-C_{s} K_{s}$.

(3) When general hospitals and specialized hospitals do not select the knowledge sharing strategy, the benefits of both groups include normal benefits and punishment for the nonsharing behavior of knowledge.

The benefit of general hospitals can be expressed as $E_{g}-\beta_{g}$.

The benefit of specialized hospitals can be expressed as $E_{s}-\beta_{s}$.

(4) When the general hospital selects the knowledge sharing strategy while the specialized hospital does not select the knowledge sharing strategy, the benefits of the general hospital include normal benefits, shared incentive, and costs, and the benefits of the specialized hospital include normal benefits, direct benefits, and punishments.

The benefit of general hospitals can be expressed as $E_{g}+\alpha_{g} \lambda_{g} K_{g}-C_{g} K_{g}$.

The benefit of specialized hospitals can be expressed as $E_{s}+\mu_{s} t_{s} K_{g}-\beta_{s}$.

Based on the above variables and benefit functions, the benefit matrix of hospital knowledge sharing under the background of Telemedicine service is shown in Table 1.
According to the above game relationship, let the probability that the general hospital selects the knowledge sharing strategy be $m$, and let the probability that the general hospital does not select the knowledge sharing strategy be $1-m$. Let the probability that the specialized hospital selects the knowledge sharing strategy be $n$, and let the probability that the specialized hospital does not select the knowledge sharing strategy be $1-n$, for $0 \leq m \leq 1,0 \leq n \leq 1$.

The equation of the benefit when the general hospital selects the knowledge sharing strategy can be expressed as

$$
\begin{aligned}
U_{m}= & n\left(E_{g}+\mu_{g} t_{g} K s+\sigma \lambda_{s} K_{s}+\alpha_{g} \lambda_{g} K_{g}-C_{g} K_{g}\right) \\
& +(1-n)\left(E_{g}+\alpha_{g} \lambda_{g} K_{g}-C_{g} K_{g}\right)_{g} .
\end{aligned}
$$

The equation of the benefit when the general hospital does not select the knowledge sharing strategy can be expressed as

$$
\left.U_{1-m}=n\left(E_{g}+\mu_{g} t_{g} K_{s}-\beta_{g}\right)+(1-n)\right)\left(E_{g}-\beta_{g}\right) .
$$

The equation of the average benefit of the general hospital can be expressed as

$$
\bar{U}_{m, 1-m}=m U_{m}+(1-m) U_{1-m} .
$$

The dynamics replication equation of the general hospital can be expressed as

$$
\begin{aligned}
F(m) & =\frac{\mathrm{d} m}{\mathrm{~d} t}=m\left(U m-\bar{U}_{m, 1-m}\right) \\
& =m\left(U_{m}-\left(m U_{m}+(1-m) U_{1-m}\right)\right) \\
& =m(1-m)\left(U_{m}-U_{1-m}\right) .
\end{aligned}
$$

Taking (1) and (2) into (4), we can obtain the following equation:

$$
F(m)=\frac{\mathrm{d} m}{\mathrm{~d} t}=m(1-m)\left(n \sigma \lambda_{s} K_{s}+a_{g} \lambda_{g} K_{g}-C_{g} K_{g}+\beta_{g}\right) .
$$

Likewise, the dynamics replication equation of the specialized hospital can be expressed as

$$
F(n)=\frac{\mathrm{d} n}{\mathrm{~d} t}=n(1-n)\left(m \sigma \lambda_{g} K_{g}+a_{s} \lambda_{s} K_{s}-C_{s} K_{s}+\beta_{s}\right) .
$$

According to the differential equations determined by (5) and (6), the evolutionary game process of hospital knowledge sharing under the background of Telemedicine service can be described. The Jacobi matrix of this evolutionary system is as follows:

$$
J=\left[\begin{array}{ll}
a & b \\
c & d
\end{array}\right]=\left[\begin{array}{cc}
(1-2 m)\left(n \sigma \lambda_{s} K_{s}+a g \lambda_{g} K_{g}-C_{g} K_{g}+\beta_{g}\right) & m(1-m) \sigma \lambda_{s} K_{s} \\
n(1-n) \sigma \lambda_{g} K_{g} & (1-2 n)\left(m \sigma \lambda_{g} K_{g}+a_{s} \lambda_{s} K_{s}-C_{s} K_{s}+\beta_{s}\right)
\end{array}\right] .
$$


TABLE 1: The benefit matrix of hospital knowledge sharing under the background of Telemedicine service.

\begin{tabular}{|c|c|c|c|}
\hline & & \multicolumn{2}{|c|}{ Specialized hospitals } \\
\hline & & Knowledge sharing & Knowledge nonsharing \\
\hline General hospitals & $\begin{array}{c}\text { Knowledge sharing } \\
\text { Knowledge nonsharing }\end{array}$ & $\begin{array}{c}E_{g}+\mu_{g} t_{g} K_{g}+\sigma \lambda_{g} K_{g}+\alpha_{g} \lambda_{g} K_{g}-C_{g} K_{g} \\
E_{s}+\mu_{s} t_{s} K_{g}+\sigma \lambda_{g} K_{g}+\alpha_{s} \lambda_{s} K_{s}-C_{s} K_{s} \\
E_{g}+\mu_{g} t_{g} K_{s}-\beta_{g} \\
E_{s}+\alpha_{s} \lambda_{s} K_{s}-C_{s} K_{s}\end{array}$ & $\begin{array}{c}E_{g}+\alpha_{g} \lambda_{g} K_{g}-C_{g} K_{g} \\
E_{s}+\mu_{s} t_{s} K_{g}-\beta_{s} \\
E_{g}-\beta_{g} \\
E_{s}-\beta_{s}\end{array}$ \\
\hline
\end{tabular}

Let $F(m)=0$; then $m^{*}=0, m^{*}=1$, or $n^{*}=\left(\left(C_{g} K_{g}-\right.\right.$ $\left.\left.a_{g} \lambda_{g} K_{g}-\beta_{g}\right) / \sigma \lambda_{s} K_{s}\right)$.

Let $F(n)=0$; then $n^{*}=0, n^{*}=1$, or $m^{*}=\left(\left(C_{s} K_{s}-\right.\right.$ $\left.\left.a_{s} \lambda_{s} K_{s}-\beta_{s}\right) / \sigma \lambda_{g} K_{g}\right)$.

The five local equilibrium points of the system can be obtained as $O(0,0), A(0,1), B(1,0), C(1,1), E\left(m^{*}, n^{*}\right)$. The value of the determinant of $J$ is det $J=a d-b c$, and the trace value of $J$ is $\operatorname{tr} J=a+d$. If $\operatorname{det} J>0$, and $\operatorname{tr} J<0$, dynamic equilibrium points are in stable state. According to the local stability analysis of Jacobi matrix, the stability analysis of each equilibrium points can be divided into the following scenarios.

Scenario 1: when $a_{g} \lambda_{g} K_{g}+\sigma \lambda_{s} K_{s}-C_{g} K_{g}+\beta_{g}>0$ and $a_{s} \lambda_{s} K_{s}+\sigma \lambda_{g} K_{g}-C_{s} K_{s}+\beta_{s}>0$. That is, the benefits when both groups select the knowledge sharing strategy are greater than those when only one player selects the knowledge sharing strategy. The local stability analysis of each equilibrium point of the system is shown in Table 2, and the dynamic evolution of the system is shown in Figure 1.

From Table 2 and Figure 1, there are two stable evolutionary strategy points in the five local equilibrium points of hospital knowledge sharing system under the background of Telemedicine service. They are point $O(0,0)$ and point $C(1,1)$; that is, both general hospitals and specialty hospitals select knowledge sharing strategy or do not select knowledge sharing strategy. The broken line $A E B$ is composed of unstable equilibrium points $A$ and $B$ and the saddle point $E$. It is the boundary of hospital knowledge sharing that converges to different evolutionary states. When the initial state of the system is in region $A O E B$, the knowledge sharing system converges to $O(0,0)$; namely, the general hospital and the specialized hospital do not select the knowledge sharing strategy. When the initial state of the system is in region $A C E B$, the knowledge sharing system converges to $C(1,1)$; namely, both the general hospital and the specialized hospital select the knowledge sharing strategy. Therefore, the greater the area of $A C E B$ in Figure 1, the greater the probability that the system will evolve to $C(1,1)$. The area is as follows:

$$
\begin{aligned}
S_{A C E B}= & \frac{1}{2}\left[1 \times\left(1-\frac{C_{g} K_{g}-a_{g} \lambda_{g} K_{g}-\beta_{g}}{\sigma \lambda_{s} K_{s}}\right)\right] \\
& +\frac{1}{2}\left[1 \times\left(1-\frac{C_{s} K_{s}-a_{s} \lambda_{s} K_{s}-\beta_{s}}{\sigma \lambda_{g} K_{g}}\right)\right] \\
= & \frac{1}{2}\left(2-\frac{C_{g} K_{g}-a_{g} \lambda_{g} K_{g}-\beta_{g}}{\sigma \lambda_{s} K_{s}}-\frac{C_{s} K_{s}-a_{s} \lambda_{s} K_{s}-\beta_{s}}{\sigma \lambda_{g} K_{g}}\right) .
\end{aligned}
$$

Scenario 2: when $a_{g} \lambda_{g} K_{g}+\sigma \lambda_{s} K_{s}-C_{g} K_{g}+\beta_{g}>0$ and $a_{s} \lambda_{s} K_{s}+\sigma \lambda_{g} K_{g}-C_{s} K_{s}+\beta_{s}<0$. Namely, the benefits of the general hospital when both the general hospital and the specialized hospital select the knowledge sharing strategy are more than the benefits when the specialized hospital selects the knowledge sharing strategy but the general hospital does not select it. Besides, the benefits of the specialized hospital when both the general hospital and the specialized hospital select the knowledge sharing strategy are less than the benefits when the general hospital selects the knowledge sharing strategy but the specialized hospital does not select it. The local stability analysis of each equilibrium point of the system is shown in Table 3, and the dynamic evolution of the system is shown in Figure 2.

Scenario 3: when $a_{g} \lambda_{g} K_{g}+\sigma \lambda_{s} K_{s}-C_{g} K_{g}+\beta_{g}<0$ and $a_{s} \lambda_{s} K_{s}+\sigma \lambda_{g} K_{g}-C_{s} K_{s}+\beta_{s}>0$. Namely, the benefits of the general hospital when both the general hospital and the specialized hospital select the knowledge sharing strategy are less than the benefits when the specialized hospital selects the knowledge sharing strategy but the general hospital does not select it. Besides, the benefits of the specialized hospital when both the general hospital and the specialized hospital select the knowledge sharing strategy are more than the benefits when the general hospital selects the knowledge sharing strategy but the specialized hospital does not select it. The local stability analysis of each equilibrium point of the system is shown in Table 3 , and the dynamic evolution of the system is shown in Figure 3.

Scenario 4: when $a_{g} \lambda_{g} K_{g}+\sigma \lambda_{s} K_{s}-C_{g} K_{g}+\beta_{g}<0$ and $a_{s} \lambda_{s} K_{s}+\sigma \lambda_{g} K_{g}-C_{s} K_{s}+\beta_{s}>0$. Namely, the benefits of the general hospital and the specialized hospital when they both select the knowledge sharing strategy are less than the benefits when one player does not select the knowledge sharing strategy but the other player selects it. The local stability analysis of each equilibrium point of the system is shown in Table 3, and the dynamic evolution of the system is shown in Figure 4.

3.2. Model Solution. According to the dynamic evolution game model above, the evolution of hospitals knowledge sharing under the background of Telemedicine service may eventually tend toward selecting the knowledge sharing strategy on both sides and may also tend toward not selecting the knowledge sharing strategy on both sides. The ultimate evolution equilibrium point and evolution path of the system depend on the benefits matrix of the game and the initial value of the related parameters. The effects of several parameters on the results of the evolutionary system will be discussed below: 
TABLe 2: Local stability analysis results of Scenario 1.

\begin{tabular}{|c|c|c|c|c|c|}
\hline Equilibrium point & $\operatorname{det} J$ & Symbol & $\operatorname{tr} J$ & Symbol & Part stability \\
\hline$O(0,0)$ & $\begin{array}{l}\left(a_{g} \lambda_{g} K_{g}-C_{g} K_{g}+\beta_{g}\right) \\
\left(a_{s} \lambda_{s} K_{s}-C_{s} K_{s}+\beta_{s}\right)\end{array}$ & + & $\begin{array}{l}\left(a_{g} \lambda_{g} K_{g}-C_{g} K_{g}+\beta_{g}\right)+ \\
\left(a_{s} \lambda_{s} K_{s}-C_{s} K_{s}+\beta_{s}\right)\end{array}$ & - & ESS \\
\hline$A(0,1)$ & $\begin{array}{l}\left(\sigma \lambda_{s} K_{s}+a_{g} \lambda_{g} K_{g}^{s}-C_{g} K_{g}+\beta_{g}\right) \\
{\left[-\left(a_{s} \lambda_{s} K_{s}-C_{s} K_{s}+\beta_{s}\right)\right]}\end{array}$ & + & $\begin{array}{l}\left(\sigma \lambda_{s} K_{s}+a_{g} \lambda_{g} K_{g}-C_{g} K_{g}+\beta_{g}\right)- \\
\left(a_{s} \lambda_{s} K_{s}-C_{s} K_{s}+\beta_{s}\right)\end{array}$ & + & Unstable \\
\hline$B(1,0)$ & $\begin{array}{l}{\left[-\left(a_{g} \lambda_{g} K_{g}-C_{g} K_{g}+\beta_{g}\right)\right]} \\
\left(\sigma \lambda_{g} K_{g}+a_{s} \lambda_{s} K_{s}-C_{s} K_{s}+\beta s\right)\end{array}$ & + & $\begin{array}{l}{\left[-\left(a_{g} \lambda_{g} K_{g}-C_{g} K_{g}+\beta_{g}\right)\right]+} \\
\left(\sigma \lambda_{g} K_{g}+a_{s} \lambda_{s} K_{s}-C_{s} K_{s}+\beta_{s}\right)\end{array}$ & + & Unstable \\
\hline$C(1,1)$ & $\begin{array}{l}{\left[-\left(\sigma \lambda_{s} K_{s}+a_{g} \lambda_{g} K_{g}-C_{g} K_{g}+\beta_{g}\right)\right] .} \\
{\left[-\left(\sigma \lambda_{g} K_{g}+a_{s} \lambda_{s} K_{s}-C_{s} K_{s}+\beta_{s}\right)\right]}\end{array}$ & + & $\begin{array}{l}-\left(\sigma \lambda_{s} K_{s}+a_{g} \lambda_{g} \mathrm{~K}_{\mathrm{g}}-\mathrm{C}_{\mathrm{g}} \mathrm{K}_{\mathrm{g}}+\beta_{g}\right)- \\
\left(\sigma \lambda_{g} K_{g}+a_{s} \lambda_{s} \mathrm{~K}_{s}-\mathrm{C}_{s} \mathrm{~K}_{s}+\beta_{s}\right)\end{array}$ & - & ESS \\
\hline$E\left(m^{*}, n^{*}\right)$ & $\begin{array}{l}(1-m)\left(a_{g} \lambda_{g} K_{g}-C_{g} K_{g}+\beta_{g}\right) \\
(1-n)\left(a_{s} \lambda_{s} K_{s}-C_{s} K_{s}+\beta_{s}\right)\end{array}$ & + & 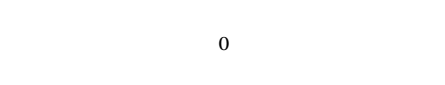 & None & Saddle point \\
\hline
\end{tabular}

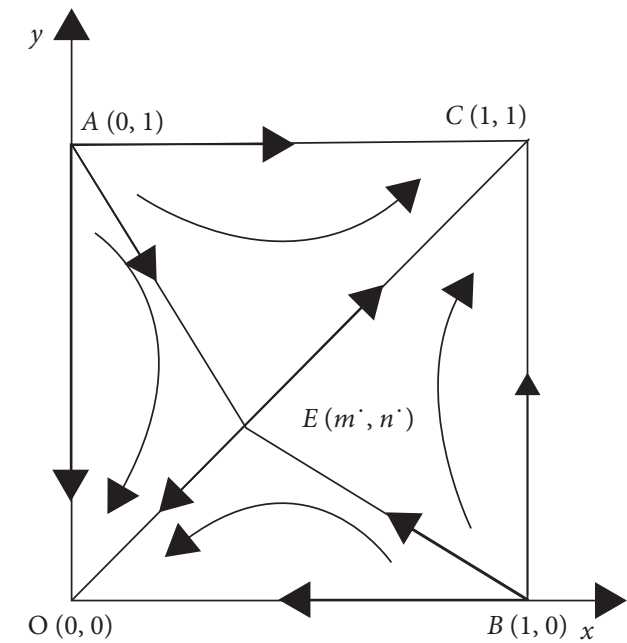

Figure 1: Dynamic evolution of the system in Scenario 1.

TABLE 3: Local stability analysis results of Scenarios 2, 3, and 4 .

\begin{tabular}{lcccccccccc}
\hline \multirow{2}{*}{ Equilibrium point } & \multicolumn{4}{c}{ Scenario 2 } & \multicolumn{3}{c}{ Scenario 3} & \multicolumn{3}{c}{ Scenario 4} \\
& $\operatorname{det} J$ & $\operatorname{tr} J$ & Part stability & $\operatorname{det} J$ & $\operatorname{tr} J$ & Part stability & $\operatorname{det} J$ & $\operatorname{tr} J$ & $\operatorname{Part}$ stability \\
\hline$O(0,0)$ & + & - & ESS & + & - & ESS & + & - & ESS \\
$A(0,1)$ & + & + & Unstable & - & Uncertainty & Saddle point & - & Uncertainty & Saddle point \\
$B(1,0)$ & - & Uncertainty & Saddle point & - & Uncertainty & Saddle point & + & + & Unstable \\
$C(1,1)$ & - & Uncertainty & Saddle point & + & + & Unstable & - & Uncertainty & Saddle point \\
\hline
\end{tabular}

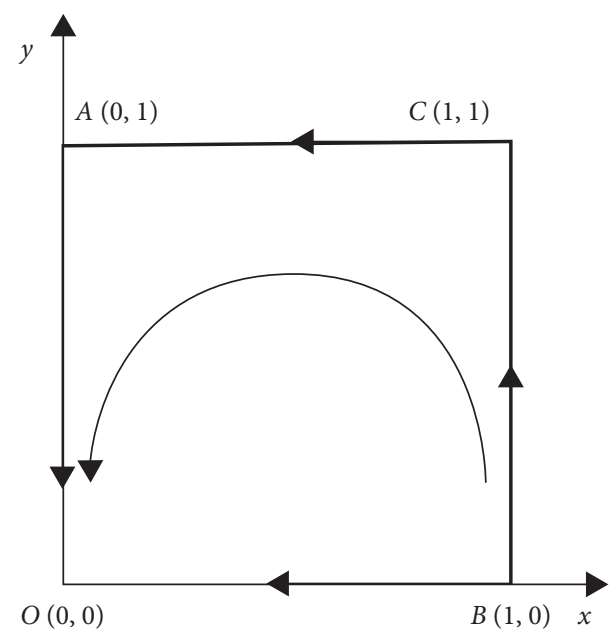

Figure 2: Dynamic evolution of the system in Scenario 2.

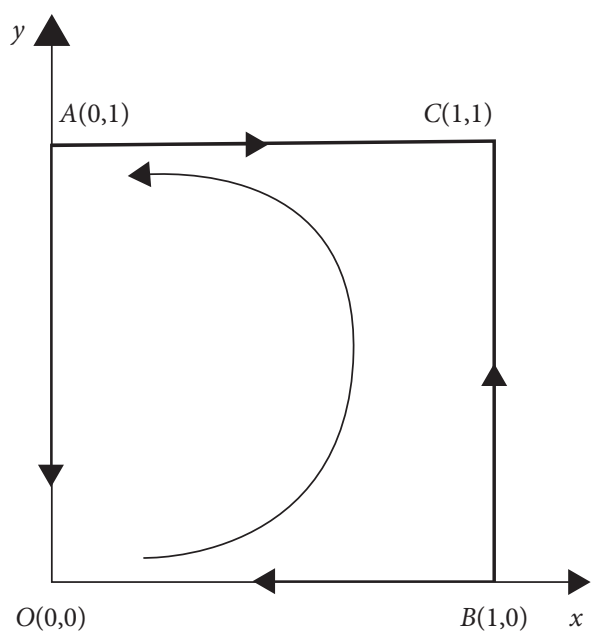

Figure 3: Dynamic evolution of the system in Scenario 3. 


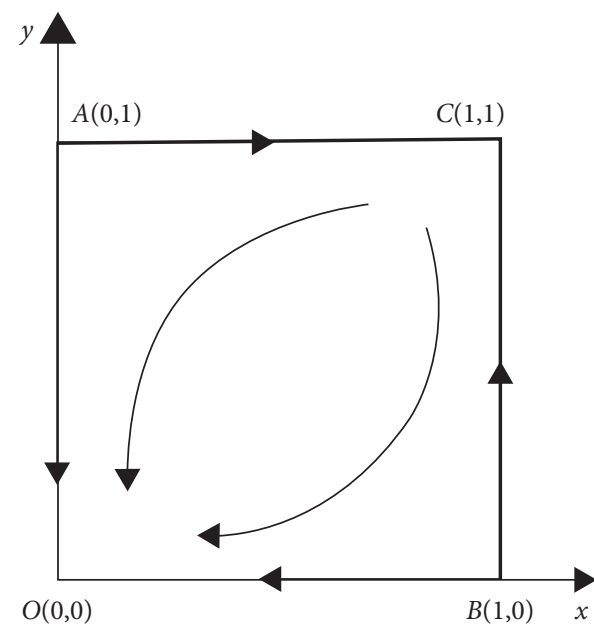

Figure 4: Dynamic evolution of the system in Scenario 4.

$$
\begin{aligned}
S_{A C E B}= & \frac{1}{2}\left[1 \times\left(1-\frac{C_{g} K_{g}-a_{g} \lambda_{g} K_{g}-\beta_{g}}{\sigma \lambda_{s} K_{s}}\right)\right] \\
& +\frac{1}{2}\left[1 \times\left(1-\frac{C_{s} K_{s}-a_{s} \lambda_{s} K_{s}-\beta s}{\sigma \lambda_{g} K_{g}}\right)\right] \\
= & \frac{1}{2}\left(2-\frac{C_{g} K_{g}-a_{g} \lambda_{g} K_{g}-\beta_{g}}{\sigma \lambda_{s} K_{s}}-\frac{C_{s} K_{s}-a_{s} \lambda_{s} K_{s}-\beta s}{\sigma \lambda_{g} K_{g}}\right) .
\end{aligned}
$$

3.2.1. Knowledge Level of the Hospital, $K_{i}$. The knowledge level of general hospital and specialized hospital is the main influence on knowledge sharing. We can see from the value $(1-m)\left(a_{g} \lambda_{g} K_{g}-C_{g} K_{g}+\beta_{g}\right) \cdot(1-n)\left(a_{s} \lambda_{s} K_{s}-\right.$ $\left.C_{s} K_{s}+\beta_{s}\right)$ of the saddle point $E\left(m^{*}, n^{*}\right)$ that, because $a_{g} \lambda_{g} K_{g}+\beta_{g}<C_{g} K_{g}$ and $a_{s} \lambda_{s} K_{s}+\beta_{s}<C_{s} K_{s}$, the value of $E\left(m^{*}, n^{*}\right)$ will gradually decreases with the increase of the knowledge level $K_{i}$, the area of the region in Figure 1 increases correspondingly, then the probability of convergence of knowledge sharing system to $C(1,1)$ increases, and the time of convergence becomes smaller. That is, the probability that general hospitals and specialized hospitals will select knowledge sharing strategy will increase, and a mutually beneficial situation will be obtained. Therefore, under the background of Telemedicine service, the general hospital and specialized hospital should strengthen the knowledge reserve, enhance the knowledge sharing advantage, and thus strengthen the knowledge sharing effect.

3.2.2. The Degree of Complementary Knowledge of Hospital, $\sigma$. Because of the different expertise of general hospitals and specialized hospitals, there are more types of cases and local common cases in general hospitals and more cases of the same type and more medical technology and equipment in specialized hospitals. If the degree of complementary knowledge of the hospital can be increased, it will be more beneficial to the knowledge sharing and the synergistic benefit in the process, thus enhancing the knowledge sharing intention of the two sides.

In the game evolution model, we can see from the value $(1-m)\left(a_{g} \lambda_{g} K_{g}-C_{g} K_{g}+\beta_{g}\right) \cdot(1-n)\left(a_{s} \lambda_{s} K_{s}-C_{s}\right.$ $\left.K_{s}+\beta_{s}\right)$ of the saddle point $E\left(m^{*}, n^{*}\right)$ that, because $a_{g} \lambda_{g} K_{g}+\beta_{g}<C_{g} K_{g}$ and $a_{s} \lambda_{s} K_{s}+\beta_{s}<C_{s} K_{s}$, the greater the degree of complementary knowledge $\sigma$, the smaller the value $E\left(m^{*}, n^{*}\right)$, the greater the area of the $A C E B$ correspondingly, and the greater the probability that and the shorter the time at which the knowledge sharing system will converge to $C(1,1)$, that is, the greater the probability that both general hospitals and specialized hospitals will select knowledge sharing strategies.

3.2.3. The Trust Degree of Hospitals, $\lambda_{i}$. It is obvious that the mutual trust of knowledge sharing can enhance the mutual knowledge sharing intention and the synergistic benefits in the process. And a good cooperative relationship can promote the continual cooperation in knowledge sharing, which has a positive impact on long-term development of Telemedicine service in China.

In the game evolution model, we can see from the value $(1-m)\left(a_{g} \lambda_{g} K_{g}-C_{g} K_{g}+\beta_{g}\right) \cdot(1-n)\left(a_{s} \lambda_{s} K_{s}-C_{s}\right.$ $\left.K_{s}+\beta_{s}\right)$ of the saddle point $E\left(m^{*}, n^{*}\right)$ that the greater the value of the trust degree $\lambda_{i}$, the smaller the value $E\left(m^{*}, n^{*}\right)$, the greater the area of the ACEB correspondingly, the greater the probability that and the shorter the time at which the knowledge sharing system will converge to $C(1,1)$, that is, the greater the probability that both general hospitals and specialized hospitals will select knowledge sharing strategies.

3.2.4. The Cost Coefficient of Knowledge Sharing in Hospital, $C_{i}$. Due to the scarcity of knowledge, the hospitals that previously had intellectual advantages would gradually lose their advantage through knowledge sharing, and knowledge sharing partners may become competitors, resulting in loss of interest of knowledge suppliers. In addition, there is certain transformation cost in the process of knowledge 
sharing duo to the hidden characteristic of knowledge. The greater the cost of knowledge sharing, the more the disadvantages to the normal transmission of knowledge sharing.

In the game evolution model, we can see from the value $(1-m)\left(a_{g} \lambda_{g} K_{g}-C_{g} K_{g}+\beta_{g}\right) \cdot(1-n)\left(a_{s} \lambda_{s} K_{s}-C_{s}\right.$ $\left.K_{s}+\beta_{s}\right)$ of the saddle point $E\left(m^{*}, n^{*}\right)$ that the greater the value of the cost coefficient of knowledge sharing, the greater the value $E\left(m^{*}, n^{*}\right)$, the smaller the area of the $A C E B$ correspondingly, and the smaller the probability that the knowledge sharing system will converge to $C(1,1)$, that is, the smaller the probability that both general hospitals and specialized hospitals will select knowledge sharing strategies.

3.2.5. The Incentive Coefficient $\alpha_{i}$ and the Punishment $\beta_{i}$. The incentive for knowledge sharing and the punishment for knowledge nonsharing play an auxiliary role in the knowledge sharing behavior of general hospitals and specialized hospitals. In the game evolution model, we can see from the value $(1-m)\left(a_{g} \lambda_{g} K_{g}-C_{g} K_{g}+\beta_{g}\right) \cdot(1-n)\left(a_{s}\right.$ $\left.\lambda_{s} K_{s}-C_{s} K_{s}+\beta_{s}\right)$ of the saddle point $E\left(m^{*}, n^{*}\right)$ that, because $a_{g} \lambda_{g} K_{g}+\beta_{g}<C_{g} K_{g}$ and $a_{s} \lambda_{s} K_{s}+\beta_{s}<C_{s} K_{s}$, the greater the value of the incentive coefficient $\alpha_{i}$ and the punishment $\beta_{i}$, the greater the area of the ACEB correspondingly in Figure 1, and the greater the probability that and the shorter the time at which the knowledge sharing system will converge to $C(1,1)$, that is, the greater the probability that both general hospitals and specialized hospitals will select knowledge sharing strategies, and ultimately a win-win result is achieved. Therefore, under the background of Telemedicine service, the probability of selecting knowledge sharing strategy can be increased by establishing reasonable incentive mechanism and punitive measure.

\section{A Case Study}

The abovementioned game model shows that that there are two evolutionary results of the behavior of hospitals in the process of knowledge sharing: both general hospitals and specialty hospitals select knowledge sharing strategy; neither general hospitals nor specialty hospitals select knowledge sharing strategy. The final equilibrium point of the evolutionary system depends on the game benefits matrix, the initial value of the parameters, and its variations. The influence of the variations of several parameters on system evolutionary results is discussed in this section, and Matlab is used to make a numerical analysis on some key parameters. The influence of different states on the evolution result of game strategy is analyzed by the graph.

We assume that the knowledge sharing probability of general hospital and specialized subject hospital is 0.5 , which is on the sidelines. The knowledge level of general hospital is 20, and the knowledge level of specialized hospital is 50 which is obviously higher than that of the general hospital. The trust degree of general hospital is 0.5 , which is higher than that of specialized hospital, 0.3. The degree of complementary knowledge of both is generally 0.5 . The cost of knowledge sharing of specialized hospitals is 0.5 which is obviously higher than that of general hospital, 0.1. The incentives and punishments of government to specialized hospitals are higher than that of general hospitals. The initial values of specific parameters are shown in Table 4.

According to the description in Section 2, we summarized all assumptions in Table 4 . We assume that $x(0 \leq x \leq 1)$ denotes the probability that general hospitals select knowledge sharing strategies, and $y(0 \leq y \leq 1)$ denotes the probability that specialized hospitals select knowledge sharing strategies. $K_{g}$ denotes the knowledge level of general hospitals, and $K_{s}$ denotes the knowledge level of specialized hospitals. $\lambda_{g}$ denotes the trust degree of general hospital, and $\lambda_{s}$ denotes the trust degree of specialized hospitals. $\sigma$ denotes the degree of complementary knowledge of general hospitals and specialized hospitals. $C_{g}$ denotes the cost coefficient of knowledge sharing in general hospitals, and $C_{s}$ denotes the cost coefficient of knowledge sharing in specialized hospitals. $\alpha \mathrm{g}$ denotes the incentive coefficient of general hospital, and $\alpha$ s denotes the incentive coefficient of specialized hospitals. $\beta_{g}$ denotes punishment of general hospital, and $\beta_{s}$ denotes punishment of specialized hospitals.

According to our hypothesis, whether the knowledge sharing of general hospitals and specialized hospitals is successful or not mainly depends on the selection strategy of specialized hospitals; the strategy selection of general hospitals in knowledge sharing is basically positive cooperation, which can be confirmed from the following graphs.

The influence of the change of knowledge level on the evolution system is shown in Figure 5. When the gap of the knowledge level between general hospitals and specialized hospitals is large, the selection strategy of specialized hospitals is evolving toward the direction of not sharing knowledge. When the knowledge level of general hospital increases to the level of the specialized hospital, the selection strategy of the specialized hospital will evolve in the direction of knowledge sharing.

The influence of the degree of complementary knowledge on the evolution system is shown in Figure 6. With the increasing of the degree of complementary knowledge, the selection strategy of specialized hospitals has been evolving toward the direction of knowledge sharing, and the speed of the evolution system has been accelerating steadily. This indicates that the higher the degree of complementary knowledge, the more conducive to enhancing the cooperation intention of mutual knowledge sharing.

The influence of the trust degree of knowledge sharing groups on the evolution system is shown in Figure 7. Only increasing the trust level of one party has some changes to the overall evolution system. However, the selection strategy of specialized hospitals still evolves in the direction of not sharing knowledge. When the trust level of both general hospitals and specialized hospitals increased, the selection strategy of specialized hospitals began to evolve toward knowledge sharing. Moreover, as the level of trust increases, the time for the evolution system to stabilize will be shortened. This shows that increasing the trust degree of 
TABLE 4: The initial values of parameters.

\begin{tabular}{lcccccc}
\hline$x$ & $y$ & $K_{g}$ & $K_{s}$ & $\lambda_{g}$ & $\lambda_{s}$ & 0.3 \\
\hline 0.5 & 0.5 & 20 & 50 & 0.5 & $\beta_{s}$ & 0.5 \\
$C_{g}$ & $C_{s}$ & $\alpha_{g}$ & $\alpha_{s}$ & $\beta_{g}$ & 10 & \\
0.1 & 0.5 & 0.2 & 0.4 & 5 & & 0.4 \\
\hline
\end{tabular}

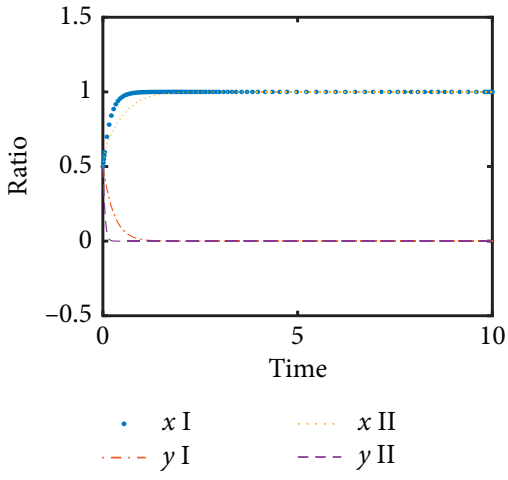

(a)

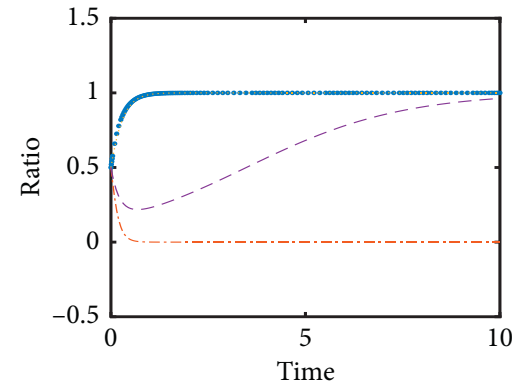

$\begin{aligned} x \mathrm{I} & \cdots \cdots x \mathrm{II} \\ --y \mathrm{I} & ---y \mathrm{II}\end{aligned}$

(b)

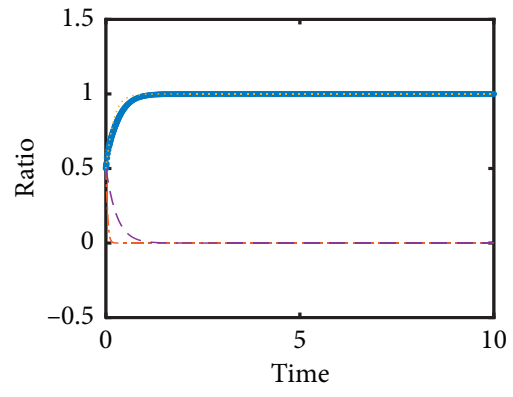

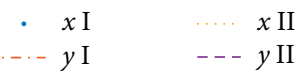

(c)

Figure 5: The influences of the change of knowledge level on the evolution system. (a) $K_{g}=50, K_{s}=100$. (b) $K_{g}=50, K_{s}=50$. (c) $K_{g}=20, K_{s}=100$.

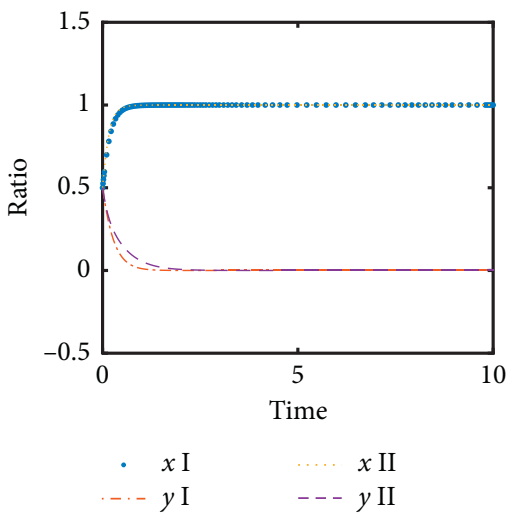

(a)

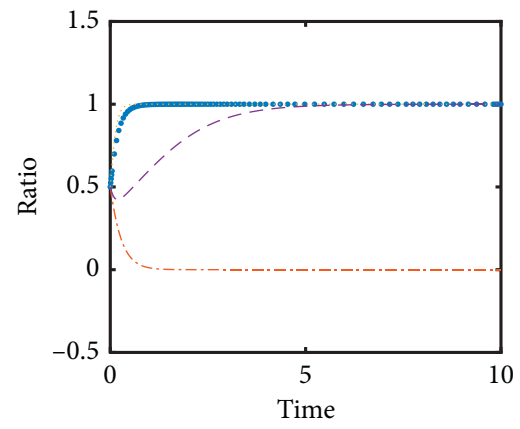

$\begin{array}{rr}\cdot \quad x \mathrm{I} \\ \cdots \cdot- & y \mathrm{I}\end{array}$

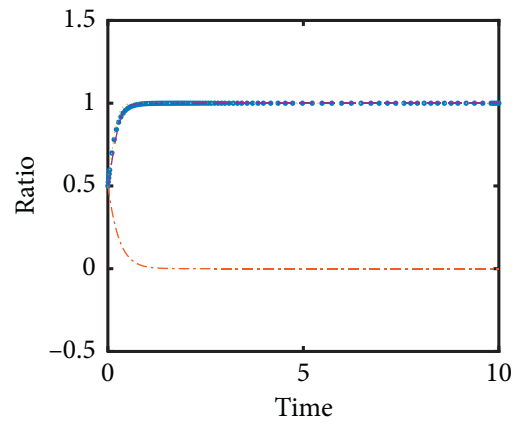

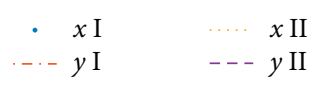

(c)

FIGURE 6: The influences of the change of the degree of complementary knowledge on the evolution system. (a) $\sigma=0.8$. (b) $\sigma=1$. (c) $\sigma=1.5$.

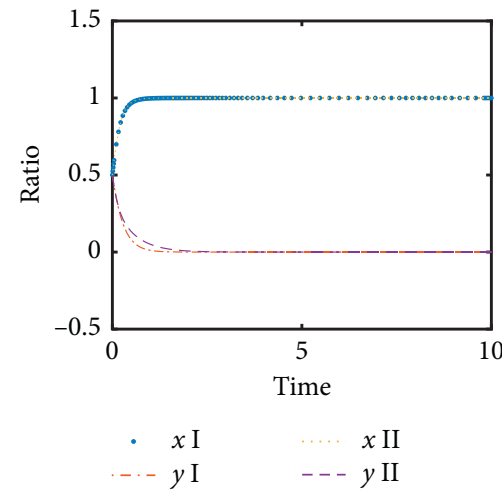

(a)

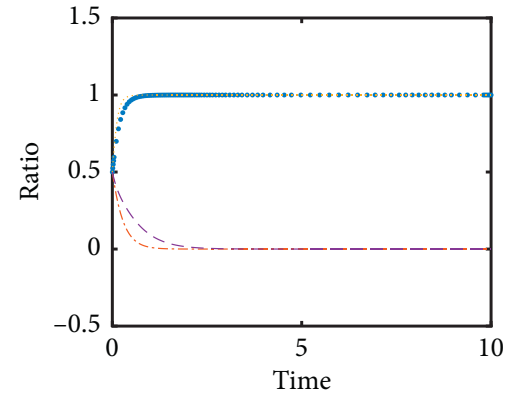

- $x \mathrm{I}$

$x$ II

(b)

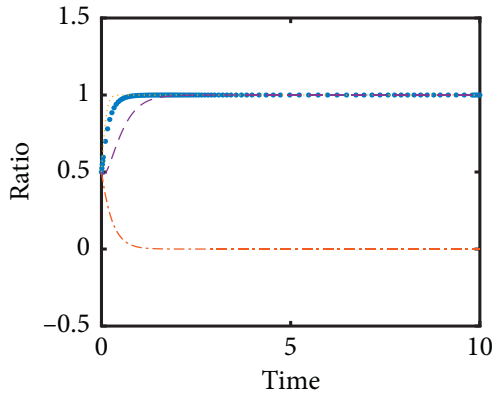

$\begin{array}{rr}x \mathrm{I} & x \mathrm{II} \\ ---y \mathrm{I} & ---y \mathrm{II}\end{array}$

(c)

FIGURE 7: The influences of the trust degree of knowledge sharing groups on the evolution system. (a) $\lambda_{g}=1, \lambda_{s}=0.3$. (b) $\lambda_{g}=0.5, \lambda_{s}=0.8$. (c) $\lambda_{g}=2, \lambda_{s}=0.8$. 


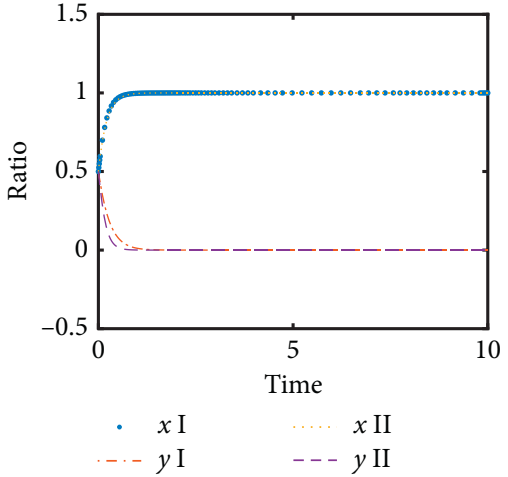

(a)

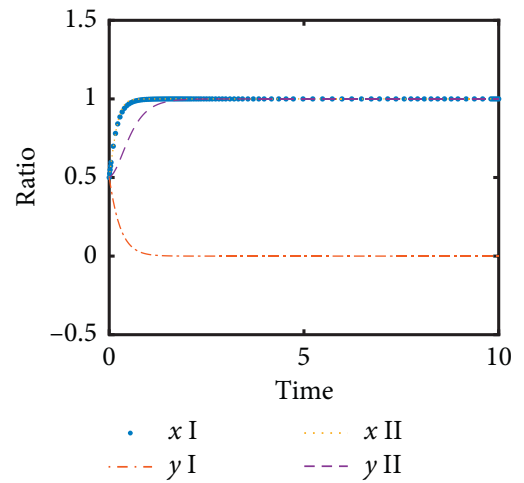

(b)

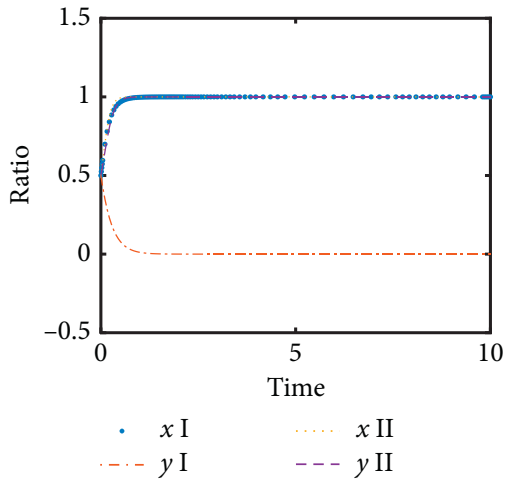

(c)

Figure 8: The influences of the cost coefficient of knowledge sharing on the evolution system. (a) $C_{g}=0.05, C_{s}=0.5$. (b) $C_{g}=0.1, C_{s}=0.3$. (c) $C_{g}=0.1, C_{s}=0.2$.

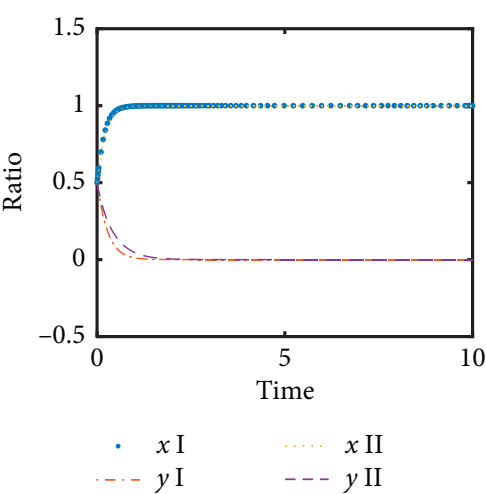

(a)

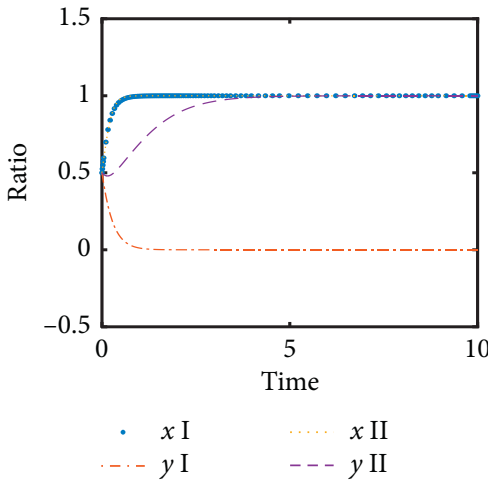

(b)

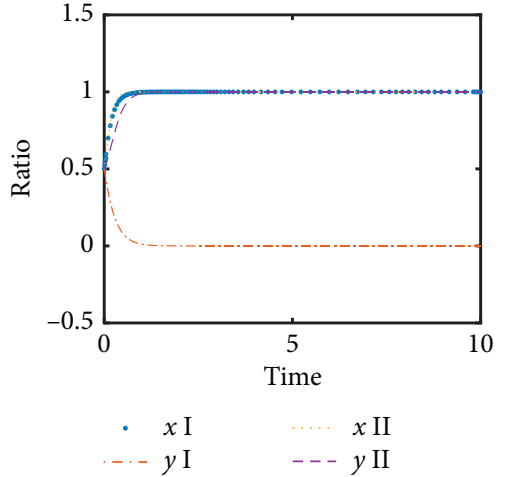

(c)

FiguRE 9: The influences of the incentive coefficient of knowledge sharing on the evolution system. (a) $\alpha_{g}=0.2, \alpha_{s}=1$. (b) $\alpha_{g}=0.2, \alpha_{s}=1.5$. (c) $\alpha_{g}=0.2, \alpha_{s}=2$.

general hospitals has a positive effect on promoting the knowledge sharing between the two groups.

The influence of the cost coefficient of knowledge sharing on the evolution system is shown in Figure 8. Reducing the cost coefficient of general hospitals has not significantly changed the overall evolution system. Reducing the cost coefficient of specialized hospitals will make the selection strategy of specialized hospitals evolve toward knowledge sharing. Besides, as the cost coefficient decreases, the time for the evolution system to stabilize the system will be shortened. This shows that if we want to promote knowledge sharing, we need to reduce the knowledge sharing cost of specialized hospitals.

The influence of the incentive coefficient of knowledge sharing on the evolution system is shown in Figure 9. When the incentive coefficient of the government $\alpha_{s}=1$, the selection strategy of specialized hospitals also evolves in the direction of not sharing knowledge. However, when the incentive coefficient $\alpha_{s}=1.5$, the selection strategy of the hospital has evolved to the direction of knowledge sharing. Furthermore, as the incentive coefficient increases, the time for the evolution system to stabilize will be shortened. This shows that the government's incentives have a positive effect on promoting knowledge sharing between the two groups.

The influence of the punishment for not sharing knowledge on the evolution system is shown in Figure 10. When the punishment of the government $\beta_{s}=15$, the selection strategy of specialized hospitals also evolves in the direction of not sharing knowledge. However, when the punishment $\beta_{s}=20$, the selection strategy of the hospital has evolved to the direction of knowledge sharing. In addition, as the punishment increases, the time for the evolution system to stabilize will be shortened. This shows that the government's punitive measures have a positive effect on promoting knowledge sharing between the two groups.

Whether general hospitals and specialized hospitals select the knowledge sharing strategy or not depends on variable conditions, such as incentive mechanism, social trends, and utility. In this section, the influencing factors of knowledge sharing between general hospitals and specialized hospitals were analyzed by numerical simulation. Through the study, we hope to improve the behavior of knowledge sharing between specialized hospitals and general hospitals in Telemedicine. 


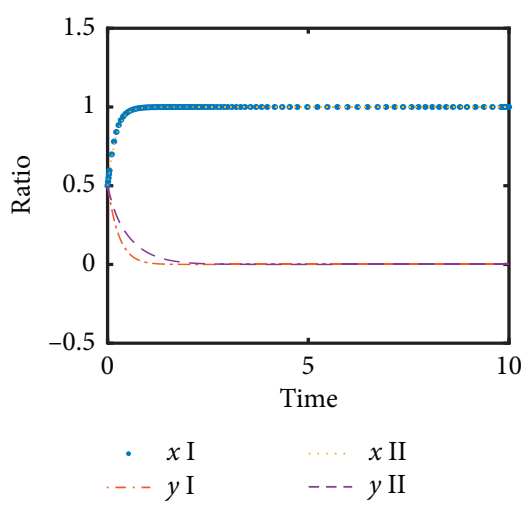

(a)

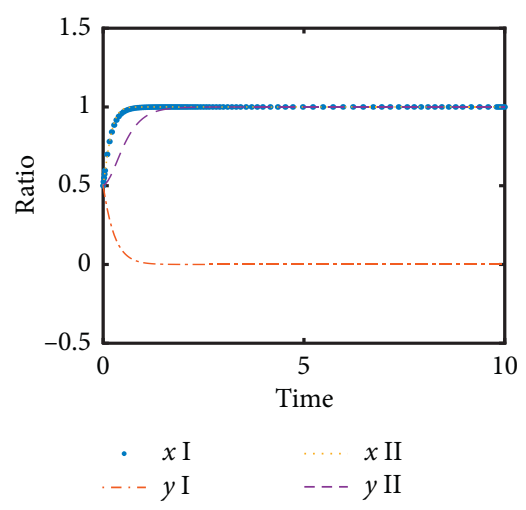

(b)

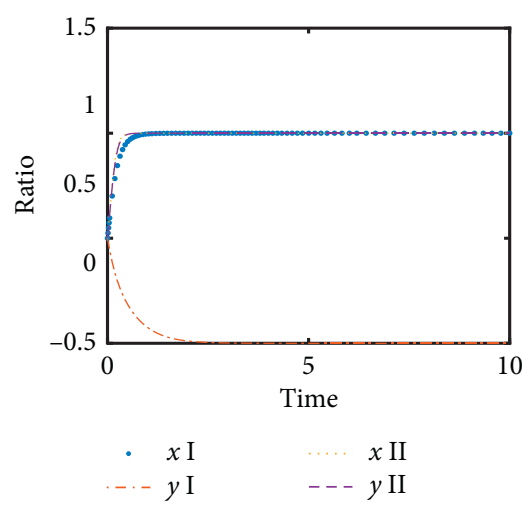

(c)

Figure 10: The influences of punishment for not sharing knowledge on the evolution system. (a) $\beta_{g}=5$, $\beta_{s}=15$. (b) $\beta_{g}=5, \beta_{s}=20$. (c) $\beta_{g}=5, \beta_{s}=25$.

In traditional group decision-making research, more consideration is given to how to reach consensus among decision-makers with different positions and preferences. Accordingly, it is more common to use the consensus theory to study relevant problems in group decision-making. Generally, a group consensus model is constructed to identify decision-makers with large differences of opinion, set up rules for opinion adjustment, and establish appropriate adjustment methods and mechanisms. In this paper, the evolutionary game theory is used to analyze the consensus decision between large groups and small groups from different perspectives or even opposing perspectives in group decision-making. This paper mainly analyzes the reasons that may affect the decision-making results of the two groups, extracts key factor indicators on this basis, and conducts evolutionary analysis to fundamentally find out the reasons for the game between large groups and small groups. In order to put forward reasonable proposals to promote consensus between the two groups, compared with consensus decision generally focusing on "how to achieve group consensus at the objective level," the research of this paper focuses on "which factors influence group consensus," so as to make the two groups choose a more consensus-reaching strategy at the subjective level.

\section{Conclusion}

In this paper, an evolutionary game method is proposed to solve the problem of knowledge sharing under the background of Telemedicine service between the large group and the small group in group decision-making problem. Next, the evolutionary model of the selection behavior of the largesmall group in combination with China's actual conditions is established. Then, the evolutionary path, evolutionary balance, and influencing factors are analyzed. It is a new study compared to the traditional group decision that distinguishes DMs into different DGs according to specific attributes.

This paper concludes that, in order to improve the knowledge sharing effect and promote the selection strategy of knowledge sharing, we need to improve the knowledge level of the large group, to increase the trust degree between two groups of knowledge sharing, to reduce the cost of knowledge sharing in the small group, and to increase the incentives and punishments for the small group. Specifically, the study mainly has the following conclusions through combining the practical problems:

(1) The small group usually has a wide range of treatment experience and numerous cases of the same type, with the absence of multiple types of cases. In order to carry out Telemedicine service in the long run and realize national medical resource sharing, the large group should firstly increase its own knowledge reserve and organize the data collection and resource integration of various local cases to improve its own advantages. This will definitely attract the small group to share knowledge and increase knowledge sharing.

(2) It is positive for government to promote and encourage the small group to collaborate with the large group in order to achieve knowledge sharing purpose. The investigation confirmed that the positive publicity of the government is conducive to increasing the trust level and positive impact on knowledge sharing. At the same time, it is helpful to improve the complementary knowledge and form a benign interaction mechanism through providing Telemedicine service training for doctors and medical staff, encouraging them to go out and participate in various professional academic activities to expand the range of knowledge sharing. It is also helpful to increase the willingness of both groups to share knowledge through creating a good knowledge sharing atmosphere in hospitals.

(3) The proper reduction of knowledge sharing cost and risk in the small group is more conducive to selecting strategy of knowledge sharing, for example, signing the contract of Telemedicine service to increase the legal protection of the small group or the protection of intellectual property rights. 
(4) The incentive mechanism can be introduced. The government could give some support to the small group that is willing to share Telemedicine service techniques and knowledge with the large group. In addition to financial support, other options include policy support in hospital evaluation or Telemedicine technical support. Thus, the initiative of participating in knowledge sharing is improved by establishing the incentive system of hospital knowledge management. There should also be some punishment for the small group unwilling to share Telemedicine services and knowledge with the large group, mainly in terms of policy and reputation.

To sum up, in the process of practical decision-making, there are differences in the subject background, knowledge, and experience between different decision-makers. Considering that there are differences between the indexes of the different decision-makers in the decision-making process, the next step is to propose a decision-making method as fair as possible for the differentiation index system of decisionmakers' preference. Moreover, the research in this paper mainly focuses on the influencing factors and evolution of consensus decision-making between the large and small groups. However, the factors that affect the large and small groups change with time and the external environment in reality. Therefore, how to distinguish these factors is also one of the directions of future research.

\section{Data Availability}

The data used to support the findings of this study are available from the corresponding author upon request.

\section{Conflicts of Interest}

The authors declare that they have no conflicts of interest regarding the publication of this paper.

\section{Acknowledgments}

This work was supported by the National Natural Science Funds of China (no. 72071152), the Natural Science Foundation Research Project of Shaanxi Province (no. 2020JQ334), the Social Science Planning Fund Project of Xi'an (no. WL215), and the Educational Reform Research Project of Xidian University.

\section{References}

[1] Z. Xu, "An error-analysis-based method for the priority of an intuitionistic preference relation in decision making," Knowledge-Based Systems, vol. 33, no. 3, pp. 173-179, 2012.

[2] Z. Xu and H. Liao, "A survey of approaches to decision making with intuitionistic fuzzy preference relations," Knowledge-Based Systems, vol. 80, no. 5, pp. 131-142, 2015.

[3] R. R. Yager, "A framework for multi-source data fusion," Information Sciences, vol. 163, no. 1-3, pp. 175-200, 2004.

[4] R. R. Yager, "Perception-based granular probabilities in risk modeling and decision making," IEEE Transactions on Fuzzy Systems, vol. 14, no. 2, pp. 329-339, 2006.
[5] Z. W. G Gon, X. X. Xu, H. H. Zhang, U. A. Ozturk, E. H. Viedma, and C. Xu, "The consensus models with interval preference opinions and their economic interpretation," Omega, vol. 55, pp. 81-90, 2015.

[6] Z. Gong, H. Zhang, J. Forrest, L. Li, and X. Xu, "Two consensus models based on the minimum cost and maximum return regarding either all individuals or one individual," European Journal of Operational Research, vol. 240, no. 1, pp. 183-192, 2015.

[7] J. Wu, L. Dai, F. Chiclana, H. Fujita, and E. Herrera-Viedma, "A minimum adjustment cost feedback mechanism based consensus model for group decision making under social network with distributed linguistic trust," Information Fusion, vol. 41, pp. 232-242, 2018.

[8] J. Wu, F. Chiclana, H. Fujita, and E. Herrera-Viedma, "A visual interaction consensus model for social network group decision making with trust propagation," Knowledge-Based Systems, vol. 122, pp. 39-50, 2017.

[9] J. Wu, F. Chiclana, and H. Liao, "Isomorphic multiplicative transitivity for intuitionistic and interval-valued fuzzy preference relations and its application in deriving their priority vectors," IEEE Transactions on Fuzzy Systems, vol. 26, no. 1, pp. 193-202, 2018.

[10] Y. Liu, C. Liang, and J. Chiclana, "A trust induced recommendation mechanism for reaching consensus in group decision making," Knowledge-Based Systems, vol. 119, pp. 221-231, 2017.

[11] B. Sun, W. Ma, and H. Zhao, "Rough set-based conflict analysis model and method over two universes," Information Sciences, vol. 372, pp. 111-125, 2016.

[12] B. Sun, W. Ma, and H. Zhao, "An approach to emergency decision making based on decision-theoretic rough set over two universes," Soft Computing, vol. 20, no. 9, pp. 3617-3628, 2016.

[13] B. Sun, W. Ma, and X. Xiao, "Three-way group decision making based on multigranulation fuzzy decision-theoretic rough set over two universes," International Journal of Approximate Reasoning, vol. 81, pp. 87-102, 2017.

[14] D.-F. Li, G.-H. Chen, and Z.-G. Huang, "Linear programming method for multiattribute group decision making using IF sets," Information Sciences, vol. 180, no. 9, pp. 1591-1609, 2010.

[15] D. F. Li and J. C. Liu, "A parameterized non-linear programming approach to solve matrix games with payoffs of I-fuzzy numbers," IEEE Transactions on Fuzzy Systems, vol. 23, no. 4, pp. 885-896, 2015.

[16] Y. Gao, Y. Du, B. Sun, R. Wang, and C. Jiang, "Tripartite evolutionary game analysis on selection behavior of transregional hospitals and patients in telemedicine system," International Journal of Computational Intelligence Systems, vol. 10, no. 1, pp. 1132-1148, 2017.

[17] G.-F. Yu, D.-F. Li, and W. Fei, "A novel method for heterogeneous multi-attribute group decision making with preference deviation," Computers \& Industrial Engineering, vol. 124, pp. 58-64, 2018.

[18] G. F. Yu, W. Fei, and D. F. Li, “A compromise-typed variable weight decision method for hybrid multi-attribute decision making," IEEE Transactions on Fuzzy Systems, vol. 41, 2018.

[19] S.-P. Wan and D.-F. Li, "Fuzzy LINMAP approach to heterogeneous MADM considering comparisons of alternatives with hesitation degrees," Omega, vol. 41, no. 6, pp. 925-940, 2013.

[20] Y. Gao, Y. Du, B. Sun, and H. Liang, "The Large-small groupbased consensus decision method and its application to 
teaching management problems," IEEE Access, vol. 7, pp. 6804-6815, 2019.

[21] B. G. Xin and M. H. Sun, "A differential oligopoly game for optimal production planning and water savings," European Journal of Operational Research, vol. 41, 2017.

[22] D. Y. Xie, F. Guan, and Q. Zhang, "A further research on S-core for interval cooperative games," International Journal of Computational Intelligence Systems, vol. 2, no. 8, pp. 307316, 2015.

[23] S. Karray and S. P. Sigué, “A game-theoretic model for copromotions: choosing a complementary versus an independent product ally," Omega, vol. 54, pp. 84-100, 2015.

[24] E. Simhon and D. Starobinski, "On the impact of information disclosure on advance reservations: a game-theoretic view," European Journal of Operational Research, vol. 267, no. 3, pp. 1075-1088, 2018.

[25] F. Meng, Q. Zhang, and Y. Wang, "Cooperative fuzzy games with a coalition structure and interval payoffs," International Journal of Computational Intelligence Systems, vol. 6, no. 3, pp. 548-558, 2013.

[26] F. Y. Meng, "Cooperative fuzzy games with convex combination form," Asia Pacific Journal of Operational Research, vol. 33, no. 1, 2016.

[27] Q. An, F. Meng, S. Ang, and X. Chen, "A new approach for fair efficiency decomposition in two-stage structure system," Operational Research, vol. 18, no. 1, pp. 257-272, 2018.

[28] S. P. Wan, L. Zhong, and J. Y. Dong, "A new method for group decision making with hesitant fuzzy preference relations based on multiplicative consistency," IEEE Transactions on Fuzzy Systems, vol. 28, no. 7, pp. 1449-1463, 2020.

[29] S. P. Wan, W. C. Zou, and J. Y. Dong, "Prospect theory based method for heterogeneous group decision making with hybrid truth degrees of alternative comparisons," Computers \& Industrial Engineering, vol. 41, 2020. 